\title{
Blue whiting protein hydrolysates stimulate GLP-1 secretion from STC-1 cells
}

\author{
S. Heffernan ${ }^{1}$, P.A. Harnedy-Rothwell ${ }^{2}$, S. Gite ${ }^{3}$, J. Whooley ${ }^{3}$, L. Giblin ${ }^{4}$, R.J. FitzGerald ${ }^{2}$ and \\ N.M. O’Brien \\ ${ }^{1}$ School of Food and Nutritional Sciences, University College Cork, Cork, Ireland, \\ ${ }^{2}$ Department of Biological Sciences, University of Limerick, Limerick, Ireland, \\ ${ }^{3}$ Bio-Marine Ingredients Ireland Ltd., Lough Egish Food Park Castleblaney, Ireland and \\ ${ }^{4}$ Teagasc Food Research Centre, Moorepark, Ireland
}

The identification of bioactive compounds from food sources that induce the feeling of fullness and may result in reduced food intake over time is currently an active area of research. Upon contact with ingested nutrients, short-term satiety hormones such as glucagonlike peptide-1 (GLP-1), cholecystokinin (CCK) and peptide YY (PYY) are secreted from enteroendocrine cells located mainly along the gastrointestinal (GI) tract and relay information to the brain to regulate appetite ${ }^{(1)}$. The ability of certain proteins to modulate GLP-1 secretion is particularly interesting considering positive reports from clinical intervention trials where food protein and protein hydrolysate consumption reduced appetite and body weight ${ }^{(2)}$. The present study was designed to assess the ability of blue whiting soluble protein hydrolysates (BWSPHs) to modulate the secretion and/or production of satiety hormones GLP-1, CCK and PYY in murine enteroendocrine STC-1 cells; to determine what impact, if any, simulated gastrointestinal digestion (SGID) has on these responses; and to determine the mechanism by which the hydrolysates mediate their satiety activity.

STC-1 cells were exposed for $4 \mathrm{~h}$ to BWSPHs and SGID BWSPHs prepared in Krebs-Ringer buffer at a final concentration of $1.0 \%$ (w/v dw). Active GLP-1 concentration was quantified using MAGPIX ${ }^{\circledR}$ software. GLP-1 precursor proglucagon, CCK and PYY mRNA transcript levels were measured using real-time polymerase chain reaction (rt-PCR). Intracellular calcium levels were mea-

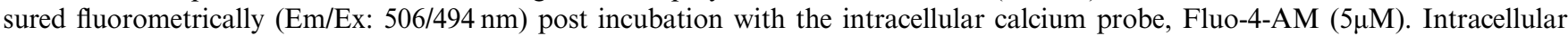
cAMP levels were assessed using a direct cAMP ELISA kit. Statistical analysis was determined using ANOVA followed by Dunnett's test (Prism 5.0, GraphPad Inc. San Diego, CA, USA). Results are expressed as mean \pm SEM of three independent trials.

All BWSPHs increased GLP-1 secretion from STC-1 cells $(2727.6 \pm 160.5-5163.8 \pm 495.6 \mathrm{pg} / \mathrm{mL})$ compared with the basal control (Krebs-Ringer buffer) $(769.7 \pm 63.5 \mathrm{pg} / \mathrm{mL})(\mathrm{p}<0.05)$. All BWSPHs also upregulated $(\mathrm{p}<0.05)$ proglucagon production $(1.5-1.9$ fold $)$ compared with the basal control. A significant increase $(\mathrm{p}<0.05)$ in intracellular calcium levels was observed after incubation with all BWSPHs (129 $\pm 7.3-151.54 \pm 9.8 \%)$ compared with the control $(100 \%)$, although none of the BWSPHs altered intracellular cAMP concentrations. The proglucagon regulatory ability of BWSPHs was lost post SGID. In addition, all BWSPHs and SGID BWSPHs induced no effect on CCK or PYY mRNA transcript levels compared with the control.

The results indicate that BWSPHs can upregulate proglucagon mRNA levels and increase secretion of active GLP-1 from STC-1 cells, possibly via intracellular calcium signalling. However, post SGID, BWSPHs lost proglucagon regulatory ability indicating that further hydrolysis of BWSPHs with GI proteases diminished hydrolysate bioactivity. These results suggest a role for BWSPH-derived peptides in satiety activity, however, these peptides may need to be encapsulated or protected by some means to avoid loss of activity during GI transit.

\section{References}

1. Egerod KL, Engelstoft MS, Grunddal KV et al. (2012) Endocrinology 153, 5782-5795.

2. Miguéns-Gómez A, Casanova-Martí À, Blay M et al. (2021) Nutrition Research Reviews 1-48. 